\title{
Establishment of Urban Real Estate Archives Information Management Platform Based on BIM Cloud Technology
}

\author{
Anqi Feng \\ School of Management, Guangdong University of Technology, Guangzhou, China \\ Email address: \\ 328873546@qq.com

\section{To cite this article:} \\ Anqi Feng. Establishment of Urban Real Estate Archives Information Management Platform Based on BIM Cloud Technology. Science \\ Innovation. Vol. 8, No. 5, 2020, pp. 127-133. doi: 10.11648/j.si.20200805.12
}

Received: October 9, 2020; Accepted: October 19, 2020; Published: October 30, 2020

\begin{abstract}
Absrtact: The modern information technology, represented by real estate file information model (BIM), cloud platform and big data, has developed rapidly in the development and management of urban real estate, and the basic framework of intelligent city (CIM) based on big data and information model has been gradually formed. There are many problems in urban real estate archives management in China, such as insufficient information, low visualization and intelligence, which can not meet the needs of various professional applications, including real estate evaluation and transaction, real estate maintenance and management, and the future needs of intelligent cities for urban real estate information sharing and intelligent management. By using BIM cloud technology, this study can solve the problem of 3D visualization and management information of real estate archives in the receiving and keeping of real estate archives. It plays an active role in realizing the intelligence of real estate archives and the construction of intelligent city in the future.
\end{abstract}

Keywords: BIM Cloud Technology, Real Estate Archives, Information Management, Collaborative Management Platform

\section{基于BIM云技术的城市房地产档案信息管理平台的建立}

冯安琪

广东工业大学管理学院, 广州, 中国

邮箱

328873546@qq.com

摘要：目前以房地产档案信息模型（BIM）、云平台和大数据为代表的现代信息技术在城市不动产开发与管理中得到迅速 的发展，以大数据和信息模型为平台的智慧城市（CIM）基本框架已逐步形成。我国城市房地产档案管理普遍存在着信息 程度不足、可视化与智能化程度低的问题，无法满足包括不动产评估交易、不动产维护与管理等多种专业应用需求，以及 未来智慧城市对城市不动产信息共享与智慧管理的需求。本研究在应用BIM云技术可以较好地解决在房地产档案的接收保 管中房地产档案三维可视化和管理信息化问题，对实现房地产档案智能化和未来智慧城市的建设具有积极推动作用。

关键词：BIM云技术，房地产档案，信息管理，协同管理平台

\section{1. 引言}

目前以房地产档案信息模型（BIM）、云平台和大数 据为代表的现代信息技术在城市不动产开发与管理中得
到迅速的发展, 以大数据和信息模型为平台的智慧城市 （CIM）基本框架已逐步形成。我国城市房地产档案管理 普遍存在着信息程度不足、可视化与智能化程度低的问题, 无法满足包括房地产评估交易、房地产维护与管理等多种 
专业应用需求, 以及未来智慧城市对城市不动产信息共享 与智慧管理的需求。

因此, 如何运用系统设计与信息化的研究方法, 将房 地产档案信息模型(BIM)技术与大数据技术结合, 提高城 市不动产管理的可视化与信息化水平, 并实现基于云平台 的不动产信息共享和协同工作, 为专业应用的智能化和智 慧城市的建设创造条件, 是当前城市土地资源信息化管理 需要解决的重要科学问题。

\section{2. 建立基于BIM技术的城市房地产信息档案管 理平台的必要性}

房地产是我国社会经济发展的重要支柱产业，房地产 档案是指房地产行政管理部门在房地产发证登记、地产 交易、房屋买卖、房屋改造、房屋拆迁和小区开发等活动 中, 经过收集、整理和鉴定等过程形成归档 保存的文字 材料、计算材料、图纸、图表和照片等各种载体内容的文 件材料, 它是房地产权属登记管理工作的重要依据[1]。

随着电子信息技术的飞速发展，云盘管、云网络、大 数据、无线传输等现代信息新技术的广泛应用, 客户群体 需求由实物案卷向电子档案转变, 特别是工程信息模型 （BIM）技术的快速应用和发展, 被称为“建筑业的第二 次革命”, 大大推进了房地产建造与管理的智能化和信息 化[2], 大量房地产项目的竣工移交都采用BIM的模型方式。 BIM技术的应用已成为国家建设“十三五”规划的重要内 容。

近年来, 尽管房地产档案管理也开展了一些信息化改 造工作, 仍然只是传统档案管理模式的一种简单数字化升 级, 用计算机、多媒体、网络等技术替代人工, 将实物档 案转换成数字信息。从档案的收集到最终的利用的过程和 结果来看, 目前的房地产档案管理还不能适应行业发展的 需求。主要体现在目前房地产档案的可视化和信息化程度 不高, 档案文件多为二维图纸和文档, 可视化的GIS地图 只能提供地理信息和三维轮廓, 缺少房地产各类属性信息 和档案信息, 基于BIM技术和云技术的协同管理平台研究 缺乏，档案资料难以同时满足三维可视化、信息集成，以 及BIM技术应用发展的要求, 档案管理在信息可视化集成 和协同工作方面的作用没有本质变化 [3]。

建立基于BIM和云技术的房地产信息档案管理平台 的意义在于:

1) 有助于充分发挥房地产档案管理部门的服务功能。 利用BIM可视化模型和云平台大数据技术, 可克服传统档 案管理的缺陷, 具有通用性强、安全性高、可视性强、支 持计算机网络查询汶览等特点。可大大提高检索效率，便 于人们查阅资料，提升房地产档案信息利用率。

通过BIM云技术所获取的房地产项目信息不仅可以 体现不同房地产信息的纵向时间关联,还可以形象化展示 出不同房地产的横向空间关联, 保障所收集的房地产档案 更全面更系统, 实现档案之间从分散独立的逻辑关系到严 密联动的逻辑关系, 在满足档案全生命周期信息化管理需 求的同时, 可大大改变传统档案收、管、用的信息化水平, 实现多参与方的信息共享和协同工作。
2）基于BIM技术的信息化档案管理系统具有很强的 分析仿真功能, 可进行多种功能模拟和指标计算, 便于更 准确的进行房地产估价和促成房地产市场交易, 可提高其 社会服务的功能和效率。

BIM 技术具有可视化和功能模拟的特征且便于进行 估价（相对于文字资料和二维的工程图纸等档案资料, BIM模型可直观地以三维的形式展现房地产项目的具体 工程形态, 便于人们更完整地了解房地产项目的空间信息 和功能, 便于信息交流和沟通; 由于BIM模型本身可以进 行工程量的统计和各种功能模拟, 如节能模拟、紧急疏散 模拟、日照模拟、热能传导模拟等, 便于进行房地产项目 的估价和不同房地产项目的偏差分析。[4]

三是档案资源具备丰富的信息属性, 房地产档案信息 化管理有助于提高档案资料的使用寿命, 保存时间长, 安 全性较强, 可满足全寿命周期房地产档案信息管理的需要, 同时能降低管理成本, 以较少的投入获得更多的资料信息, 取得更大的经济效益和社会效益。

目前国内的一些工程档案管理机构 (如上海浦东建筑 档案馆、珠海城市建筑档案馆已经开展了基于BIM技术的 房地产档案管理研究, 取得了一些研究成果 [5]。但与建筑 工程档案管理，房地产档案信息管理的“收、管、用”复杂 程度更高, 如何系统性地解决基于BIM可视化及云平台技 术的房地产档案与BIM模型的标准化问题、模型与资料关 联检索信息化问题, 可视化档案的接收、保管和利用模式 的研究问题? 如何利用BIM模型和云平台, 将时间、空间 及其他条件可形成大数据网络, 帮助不同的用户（业主、 档案管理部门、政府相关机构及市场的相关主体）可通过 云平台和大数据访问BIM相关房地产模型和信息, 实现信 息共享和协同工作, 提高房地产档案的利用率和应用功能, 满足房地产开发、物业管理、房地产估价和市场交易、城 市更新等市场发展的需要? 能够借鉴的经验是远远不够 的。

因此实现房地产档案的可视化、信息化集成和房地产 信息的实时采集、适应以BIM技术为代表的房地产建设管 理信息化技术的发展, BIM云平台和大数据等信息化手段 提升房地产档案信息的存、管、用信息化水平, 促进房地 产交易市场房地产档案的利用效率，满足未来城市更新与 智慧城市建设和发展的需要, 成为目前房地产档案信息化 管理需要解决的关键问题。

\section{3. 基于BIM云技术的城市房地产信息档案管理 平台的系统设计与实现方法}

\section{1. 基于BIM技术的城市房地产信息档案管理平台的系 统功能设计}

在构建基于BIM技术的房地产信息档案管理平台的 最终目的是将正确的信息在正确的时间提供给正确的用 户，利用BIM模型实现信息可视化和集成，利用云平台和 客户端开发, 实现各参与方收、管、用的协同工作。根据 我们前期调研和专家讨论, 我们设计的基于BIM技术的房 地产信息档案管理平台架构与功能如图1所示。 


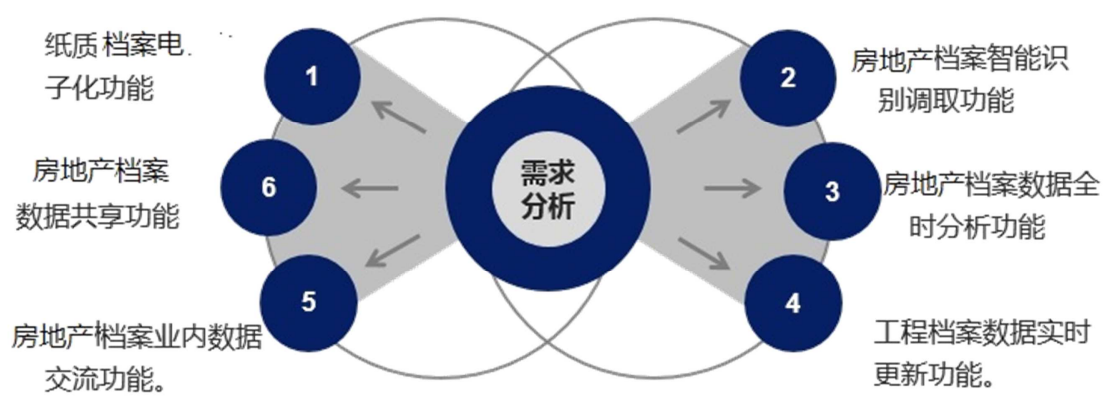

图1 基于BIM技术的房地产信息档案管理平台的功能图。

\section{2. 基于BIM技术的房地产信息档案管理平台的系统流} 程设计

\subsection{1. 基本要求}

1）遵循传统房地产档案与BIM档案并存的原则, 采 用BIM模型档案移交与传统档案移交两种存档模式共同 管理房地产信息档案。[9]

2)设置基于BIM档案信息管理的归档标准，其中传统 档案移交依据标准《建设档案文件归档整理规范》 GB/T50328-2014[6]对归档格式进行统一要求; BIM档案信 息标准依据项目研究成果的规定, 对数据接口、命名规则、 模型深度根据项目实际情况做规定。[10]

3)完成不同形式房地产档案的搜集后, 依据所规定的 代码标识、格式与载体对所有文件进行收集、整理、编码、 录入的工作。[11]

4)移交方依据规定的BIM房地产档案管理要求, 自检 文件的质量, 自检合格后方可送入档案馆进行预验收质量 合格; 其中进行编码录入后，BIM信息化管理技术可根据 数据交换、云计算等技术半自动检验提交材料的质量。[12]

5)待自检、验收合格后，依据所编制好的标准，关联 BIM档案的内部文件、传统档案文件、电子档案, 实现三 者的联动。

6)在完成BIM档案、传统档案与电子文档的相关关联 后, 即形成了基于BIM为中心的信息化移交文件, 再将档 案资料入库, 完成了整个以BIM技术为中心的档案接收流 程。

7)基于全生命周期动态的管理原则，在房地产进行较 大改造、报废拆除等较大变动后, 新的竣工档案应按照上 面顺序流程重新添加到该房地产档案中。

\subsection{2. 流程设计}

据国家相关规范和标准[7], 分析出了传统档案、BIM 移交档案及相关电子档案的移交标准, 在BIM档案移交标 准中规定了在不同阶段不同专业所用到BIM技术的数据 接口、命名规则、模型深度、编码要求等等。在上述研究 工作基础上, 可实现对已有传统BIM档案管理流程的拓展, 建立基于 BIM技术的新型房地产档案资料信息化移交模 式如下图所示:

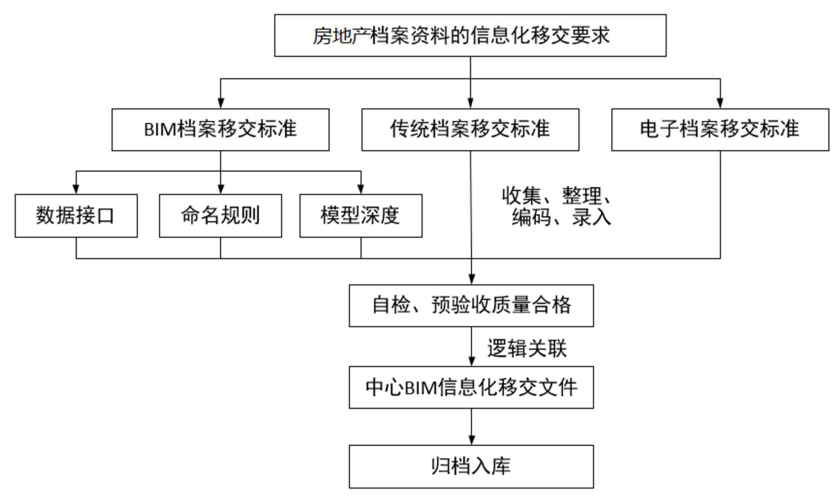

图2 房地产资料档案信息化的管理流程。

\subsection{3. 技术方案设计}

首先根据相关构件命名标准和模型命名标准建立 BIM模型, 并对相关的房地产档案资料进行收集、分类整 理和编码后, 可通过Autodesk Navisworks Manage等软件 对资料的录入实现模型与房地产文件之间的关联。具体流 程如下:

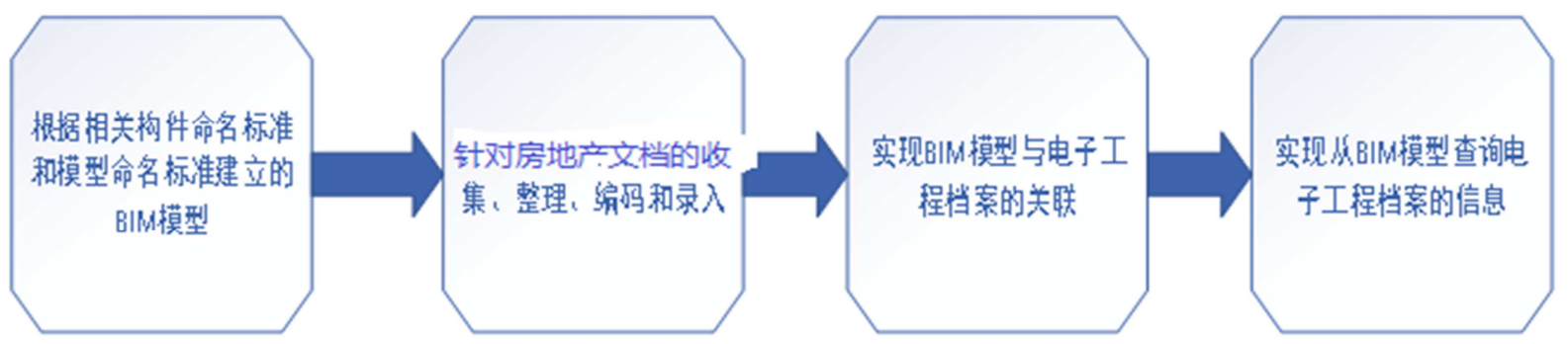

图3 方案实施流程。

云端解决方案可以通过Autodesk BIM 云平台实现 BIM模型和房地产档案文件之间的管理。以BIM 360 Docs
平台为例, 其为基于云架构的BIM数据集中存储系统 [8], 借助该系统, 才队用户能方便地访问存储在云端的BIM数 
据, 所有的访问均受权限控制, 系统提供基于web的数据 汶览方式, 降BIM数据的访问门槛。

利用BIM 360 Docs可以发布、管理、审查、标记和批 准最新的施工图文档集合,支持PDF、DWF、RVT、IFC和
DWG文件, 可以从DWG、PDF和RVT中提取图纸, 包括PDF 标题栏, 还为PDF添加了标记功能, 以及状态更改和文档 附件; 同时允许2D图纸比较。

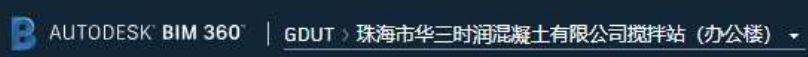

\section{并 Document Management 文使 问题}

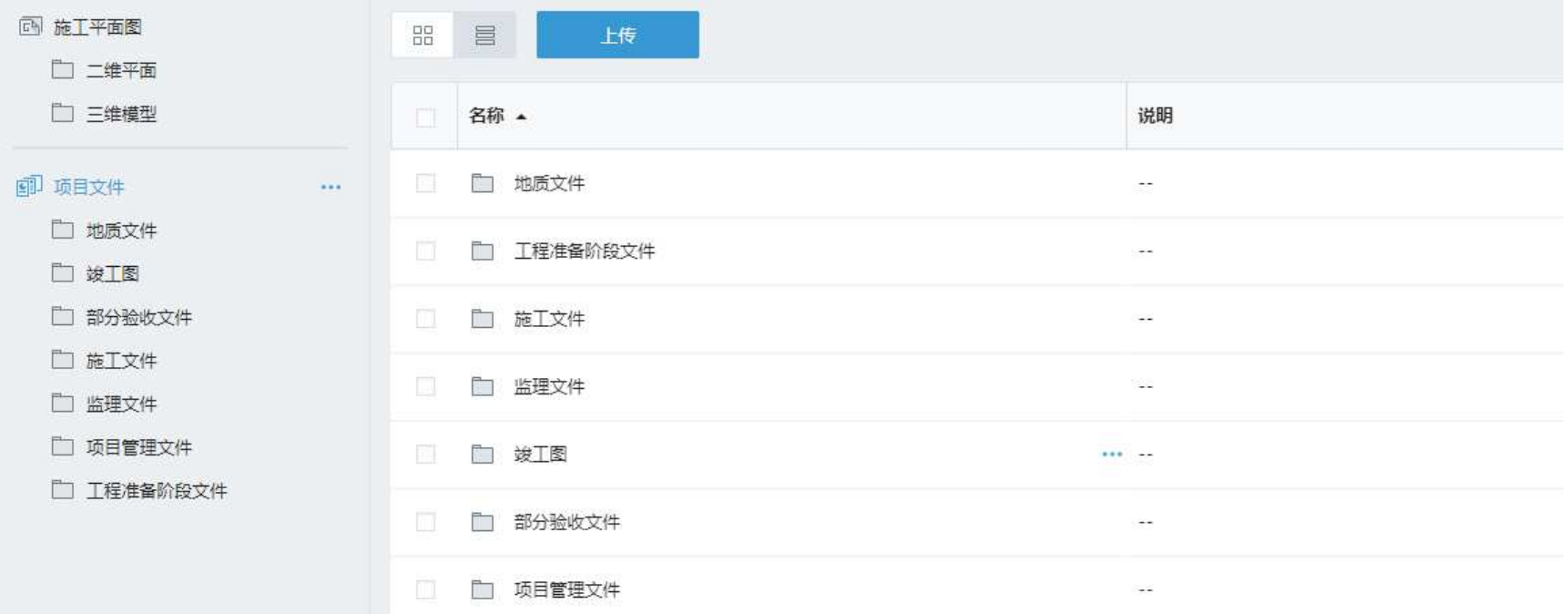

图4 分类文件集与对应信息化档案清单存储路径。

\begin{tabular}{|c|c|c|c|c|c|c|c|c|c|}
\hline \multirow{2}{*}{ 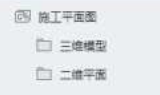 } & & $\begin{array}{l}98 \\
9\end{array}$ & \multirow[b]{2}{*}{ 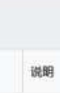 } & \multicolumn{4}{|c|}{ 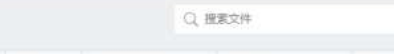 } & \multirow{2}{*}{ कि } & \multirow{2}{*}{$\frac{1}{0}$} \\
\hline & & - & & 酶本 & 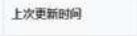 & 更絃 & 配 & & \\
\hline \multirow{7}{*}{ 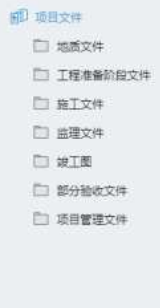 } & \multirow[t]{7}{*}{... } & 口曰口酘神 & -. & .. & 2018 年6月13日下- & Ellison Koong & .. & -. & \\
\hline & & $\square$ 口 I I & $\cdots \cdots$ & & 2018 年6月13日上 & Ellson Kong & & 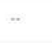 & \\
\hline & & 口曰口耓文神 & . & -. & 2018 年 6 月 18 日上 & Ellson Kong & - & -. & \\
\hline & & 17 口此理件 & - & 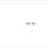 & 2018 年 6 月 13 日上. & Ellson Kong & - & - & \\
\hline & & 口曰口垠现 & - & - & 2018 年6月13日上 & Ellson Kong & - & - & \\
\hline & & 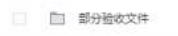 & -. & & 2018年6月13日上 & Ellson Kong & & & \\
\hline & & 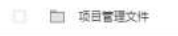 & $-\cdot$ & -. & 2018年6月13日上 & Elison Kong & -. & - & \\
\hline
\end{tabular}

图5 具体档案文件上传分类档案材料信息。 


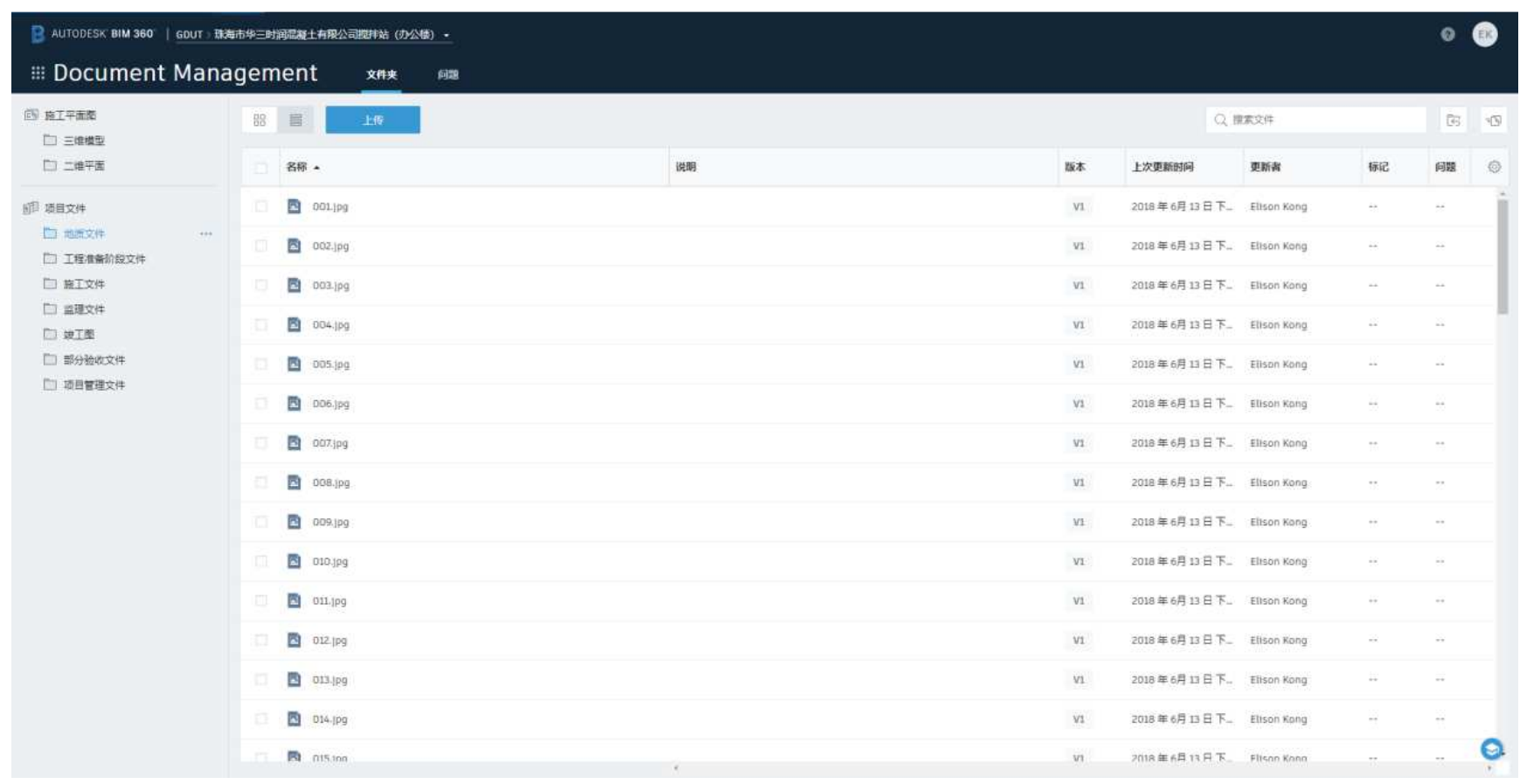

图6 上传信息具体档案记录。

\section{4. 案例应用情况}

以珠海市某房地产项目档案管理为例, 介绍该平台的 工程档案上传及档案管理功能模块的应用

\section{1. 档案文件的上传管理模块}

按照平台的规范要求建完模后将其上传到BIM云平 台, 上传界面如图7:

\section{2. 档案收取与多用户协同管理}

项目管理员可根据具体的房地产需求设定收取档案 文件的类型和路径，管理和查询上传相关项目文件信息。

目前系统平台可支持符合目前信息化规范的各种类 型项目电子文档、包括设计数据和所有其它文档类型, 包 括照片、报告、明细表、预算、要求文档等。

同时还可支持文件分层管理，创建项目的分部分项房 地产的文件夹; 可以重命名和移动文件夹, 接受通知并访 问项目交付日志, 满足日后档案类型扩展和更新需要。

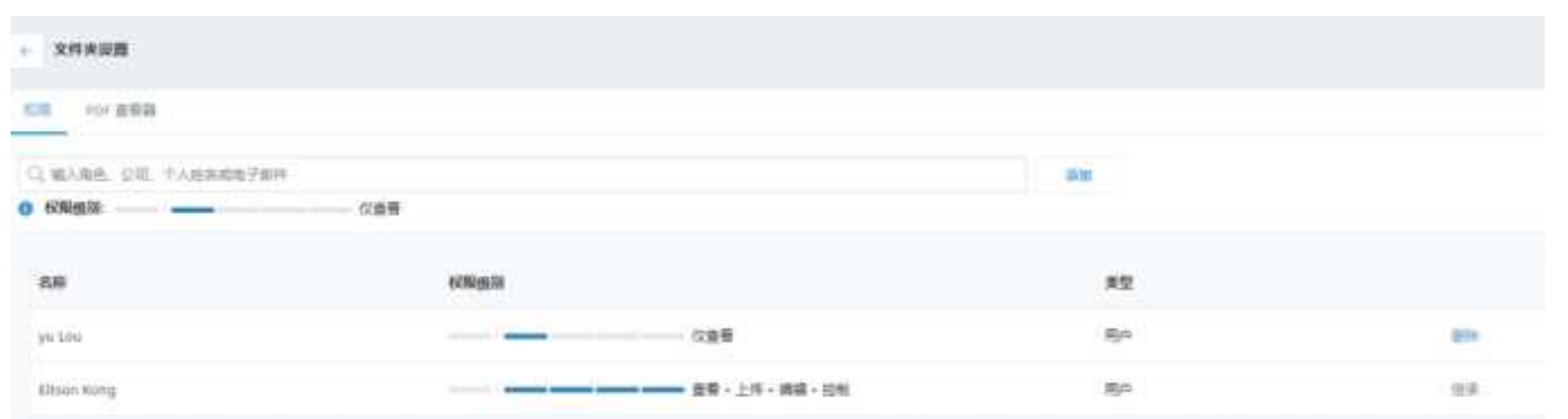

图7 多用户协同管理与权限分配。

在系统中，可邀请成员加入协同项目。在成员被邀请 参加该项目之后, 项目管理员随后将成员按角色, 添加到 文件夹中。

系统一共分配五个权限级别, 项目管理员对成员设置 访问权限。权限允许成员查看、上传、编辑或控制文件夹
中的文档。通过建立成员资格和权限, 项目管理员现在可 以为项目设置协同工作流程。在通过系统用户界面设置按 钮访问项目管理页面后, 项目管理员可以更改协同工作流 程，根据对应权限开展不同应用。 


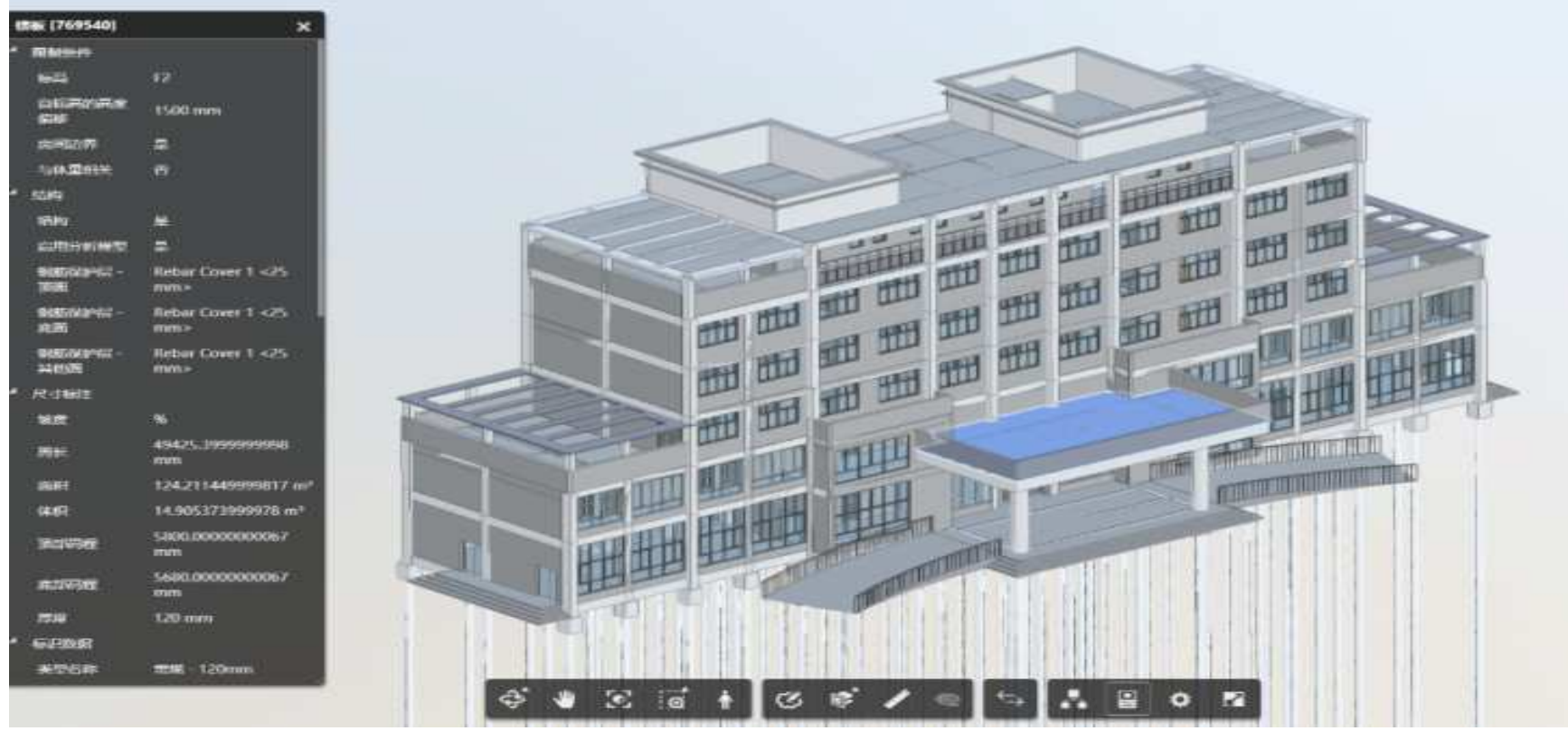

图8 不同用户可在相应权限内共享可视化档案信息。
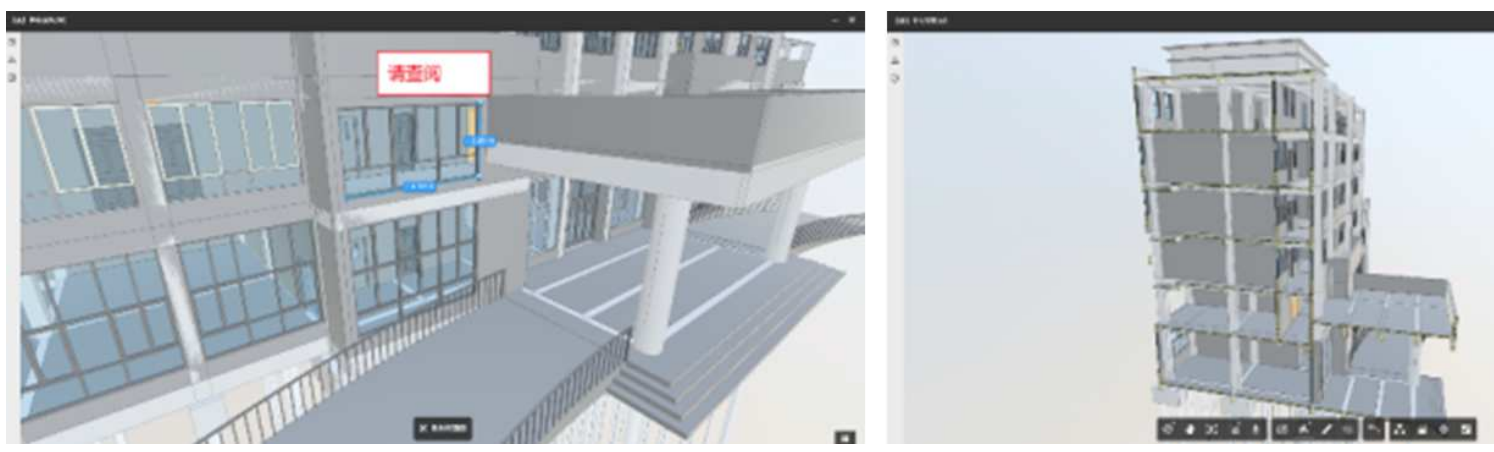

图9 系统提供用户分权限提供测量与档案资料标注功能, 提高档案应用功能。
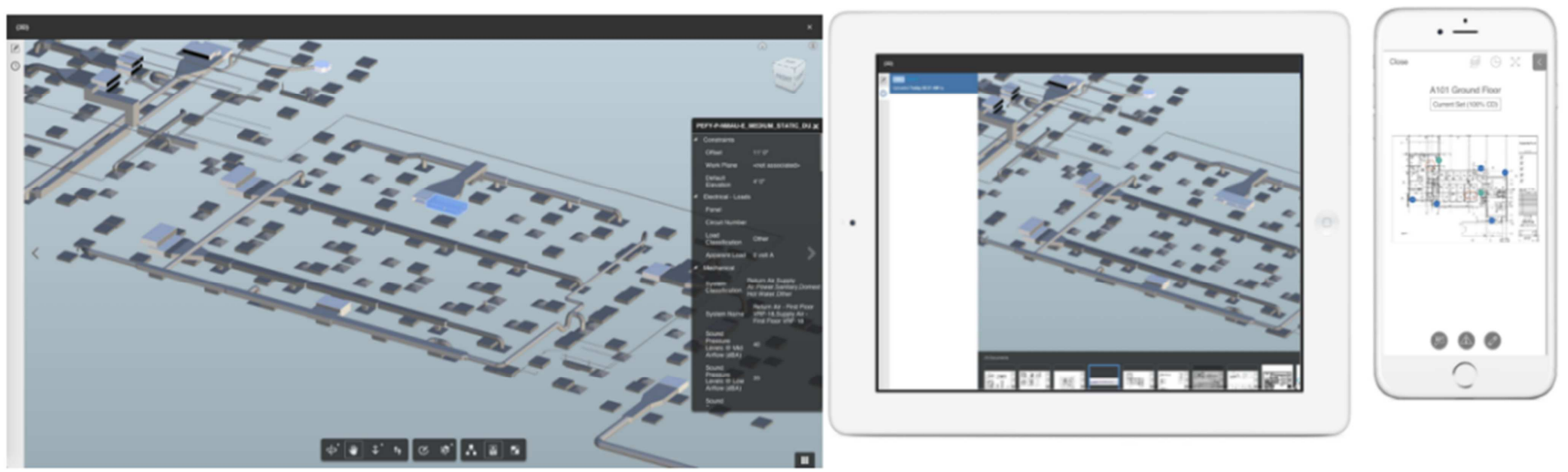

图10 利用云平台客户端APP, 用户实现远程同步上传和档案访问。

\section{5. 结论和展望}

系统开发和应用的结果表明:

利用建筑信息模型（BIM）技术，可以实现房地产档 案管理可视化和信息集成（时间、空间及工程信息等）的 目标。
将BIM模型与云技术结合建立的BIM云房地产档案 管理平台，通过权限设定和APP开发可实现不同的用户 （如业主、档案管理部门、政府相关机构等不同市场的相 关主体和用户) 可通过云平台和大数据访问BIM相关房地 产模型和信息，实现信息共享和协同工作，提高房地产档 案的利用率和应用功能。

通过BIM标准化研究和BIM云平台系统设计和模块 设计, 可以较好地解决房地产档案标准化、模型与资料关 
联检索, 实现可视化档案的接收、保管和利用等关键问题。 [13]。

展望: $\mathrm{BIM}$ 技术和云平台大数据, 特别是 $5 \mathrm{G}$ 技术的迅 猛发展必将推动我国智慧城市建设。[14]基于BIM云技术 的房地产档案协同管理平台的研究和应用, 实现了房地产 档案的可视化、信息化集成和房地产信息的实时采集、提 升房地产档案信息的存、管、用信息化水平，促进房地产 交易市场房地产档案的利用效率，[15]适应了BIM技术为 代表的城市房地产物的数字化、网络化建设发展对档案管 理的新需求, 必将全面提升城市建设档案管理的信息和智 能化发展, 可为智慧城市其他数字管理平台提供了城市基 础数据和服务。

\section{参考文献}

[1] 颜菲. 信息网络平台在房地产档案管理中的运用[J].房地产 档案,2018(07)。

[2] 杨建林.智慧城市背景下的房地产档案信息化建设[J].房地 产档案,2018(07):19-20.

[3] 沈银娇.信息化背景下房地产档案信息化管理存在的问题 及对策[J].房地产档案,2018(08)。

[4] 巩玉静.基于BIM竣工模型归档应用可行性研究方法 [J]. 智 库时代,2018(22):125-128.

[5]吕向荣. 北京建设房地产BIM档案接收必要性探讨 [J]. 房地 产档案,2018(02):12-14.
[6] GB/T50328-2014《建设档案文件归档整理规范》中华人民 共和国住房和城乡建设部2014年。

[7] GB/T51212-2016建筑信息模型应用统一标准中华人民共和 国住房和城乡建设部2016年。

[8] 杨继东.基于BIM技术的三维房地产档案接收保管和利用 模式研究[J].数字与缩微影像,2017(03):11-15.

[9] 高东东. 房地产信息模型与房地产项目管理数据的关联及 应用研究[D].深圳大学,2017.

[10] 杨镇宇,周佶.BIM云平台的应用优势与在实际运用中的局 限性[J].山西房地产,2016,42(35):255-257.

[11] 孙云萍.大数据时代下房地产档案管理信息化面临的挑战 和途径探索 [J].房地产档案,2016(06):12-14.

[12] 张肖千.不动产档案集约化管理研究[D]. 苏州大学,2016.

[13] Steven F. Mount. New Wine in Old Bottles: Has the Definition of 'Real Estate Assets' Been Expanded for Real Estate Investment Trusts?[J]. Tax Management Memorandum,2013(1):383-389.

[14] 余卫明.境外不动产登记的三种模式[J].国土资源导刊, 2014 (9) : 65-66.

[15] Ball M. Critical commentary. Cities and housing markets: Changes and continuities in the aftermath of the 2007-08 world financial crisis[J].UrbanStudies,2010:931-944. 